\title{
Efeito de fatores ambientais sobre escores de avaliação visual à desmama e estimativa de parâmetros genéticos, para bezerros da raça charolês
}

\author{
Effects of environmental factors on visual scores at weaning and genetic \\ parameters for charolais calves
}

\author{
Carlos Junior Kippert ${ }^{1}$ Paulo Roberto Nogara Rorato ${ }^{2}$ Leonardo Talavera Campos ${ }^{3}$ \\ Arione Augusti Boligon ${ }^{4}$ Tomas Weber ${ }^{4}$ Diego Ghedini Gheller $^{4}$ Jader Silva Lopes $^{4}$
}

\section{RESUMO}

Para avaliar a influência de alguns fatores de ambiente sobre escores de avaliação visual à desmama em animais da raça Charolês e estimar parâmetros genéticos, foram utilizadas informações coletadas pelo PROMEBO durante os anos de 1994 a 2002. As características estudadas foram os escores de conformação $(C)$, precocidade $(P)$, musculatura $(M)$ e tamanho $(T)$ à desmama. Inicialmente foi realizada uma análise de variância, para verificar quais fatores apresentavam efeito significativo sobre as características em estudo. O modelo considerou como fixo, o efeito de grupo de contemporâneos (GC), constituído por fazenda, sexo, estação $e$ ano de nascimento e, como covariáveis, a idade da vaca ao parto (IV), a idade do bezerro à desmama (ID) e a data juliana de nascimento $(\mathrm{DJ})$, além do efeito residual, como aleatório. Todos os efeitos incluídos no modelo foram significativos para os escores visuais $(P<0,05)$, exceto $D J$, para $M, P$ e C. A partir das médias ajustadas de $C, P, M$ e T, foram estimadas equações de regressão para $I V$, ID e DJ. Observou-se que vacas que pariram aos 7-8 anos tiveram bezerros com escores visuais maiores. Com relação à idade à desmama, verificou-se que animais desmamados mais tardiamente receberam Escores Visuais mais elevados. Com relação à data juliana de nascimento, constatou-se que bezerros nascidos em junho foram favorecidos em seus escores, quando comparados com animais nascidos em dezembro ou janeiro. Os parâmetros genéticos foram estimados pelo método da Máxima Verossimilhança Restrita livre de derivada, utilizando um modelo animal, considerando como fixo o efeito de GC e, como covariáveis, $I V$, ID e DJ e, como aleatórios, os efeitos genéticos aditivos diretos e maternos e o residual. As estimativas de herdabilidade encontradas e respectivos erros padrões, para os efeitos genéticos diretos, foram 0,37 (0,09), 0,35 (0,09), 0,33 (0,09),e
0,27 (0,09), respectivamente, para $C, M, P$ e T. Os resultados evidenciam que as avaliações para escores visuais devem levar em consideração os efeitos fixos estudados e existe a possibilidade de inserção destas características em programas de melhoramento genético, desde que ocorra redução do componente de variância ambiental, através de um melhor controle do ambiente.

Palavras-chave: coeficientes de herdabilidade direta e materna, componentes de variância, características de conformação, precocidade, musculatura $e$ tamanho.

\section{ABSTRACT}

To evaluate the influence of environmental factors on visual scores at weaning on Charolais breed animals, and to estimate genetic parameters, it were used records collected by PROMEBO from 1994 to 2002. The traits evaluated were the visual scores for conformation $(C)$, precocity $(P)$, muscling $(M)$ and size $(T)$ at weaning. First of all, it was performed an analyse of variance to verify wich environmental effects were significant on the studied characteristics. The model used considered, as fixed, the effect of contemporaneous group (GC), as covariables, the effects of age of the dam at parturition (IV), age at weaning (ID) and Julian date of birth (DJ) and the residual effect. All the variables included in the model affected significantly visual scores $(P<0,05)$, except $D J$, for $M, P$ and $C$. From the adjusted averages for $C, P, M$ and $T$ it were adjusted regression equations for $I V, I D$ and $D J$. It was observed that cows freshening between 7 and 8 years produced calves with larger scores. In relation to the weaning age, it was observed that the animals weaned later presented more elevated visual scores. In relation to the Julian date of born, it was verified that

\footnotetext{
${ }^{1}$ Curso de Pós-graduação em Zootecnia, Mestrado, Universidade Federal de Santa Maria (UFSM), Santa Maria, RS, Brasil. E- mail: kippert1@hotmail.com. Autor para corresondência.

${ }^{2}$ Departamento. de Zootecnia, UFSM, Santa Maria, RS, Brasil. E- mail: rorato@smail.ufsm.br

${ }^{3}$ Coordenador Técnico do PROMEBO-ANC, Pelotas, RS, Brasil.

${ }^{4}$ Curso de Zootecnia, UFSM, Santa Maria, RS, Brasil.
} 
calves born in June were favored in their scores, when compared with animal that born in December and January. The genetic parameters were estimated by Restricted Maximum Likelihood Method, using an animal model considering as fixed the effect of GC and, as covariables, IV, ID and DJ and, as random, the additive genetic direct and maternal and the residual effects. The heritability estimated for genetic direct effect and standard errors were .37 (.09), .35 (.09), .33 (.09) and .27 (.09), respectively, for $C, M, P$ and $T$. These results suggest that the evaluation for visual scores, must to considerate the studied fixed effects, and that it is possible to include these characteristics in breeding programs.

Key words: conformation, precocity, muscling and size characteriratics, direct and maternal heritability coefficients, variance components.

\section{INTRODUÇÃO}

Em bovinos de corte, a seleção genética de animais para as características de peso e ganho de peso é de fundamental importância e sua adoção, por certo, promove benefícios econômicos. A tendência atual de mercado é buscar animais precoces, os quais são mais viáveis economicamente, pois permanecem menos tempo nas pastagens ou confinamentos, encurtando o ciclo de produção. Desta forma, melhora a eficiência da atividade, além de prover um rápido retorno econômico ao produtor, aumentando seu lucro. A seleção somente por pesos e ganhos de peso a determinadas idades pode se mostrar insuficiente para atingir este objetivo, já que não abrange todo o potencial genético dos animais. Observa-se na raça Charolês, animais com elevado peso adulto, sendo esta uma característica inerente à raça como constatado por KIPPERT et al. (2004).

Sabe-se que o peso ao nascer, à desmama e ao sobreano, apresentam correlação alta e positiva, entre si e que a seleção para elevar um destes, promoverá também o aumento dos outros pesos (FERREIRA et al.,1999). Diante disto, teremos ao longo dos anos, animais com maior peso e, conseqüentemente, com maior exigência de mantença (NRC, 1996). A região sul do Brasil possui inverno com baixas temperaturas e poucas horas de insolação, o que limita o crescimento das pastagens naturais. Desta forma, animais de grande porte criados neste ambiente, podem não ter suas exigências nutricionais supridas.

Nesta lógica, estabeleceu-se o uso de escores visuais, que se baseiam numa escala de um a cinco para algumas características medidas visualmente, não como critério absoluto, mas como uma variável que auxilia na tomada de decisões em um programa de melhoramento genético. A inclusão de escores de avaliação visual nestes programas é uma alternativa para melhorar a produção de carne, a qualidade da carcaça, a precocidade de terminação e o rendimento no tamanho corporal, com a vantagem de ter baixo custo de implementação (CAMPOS \& CARDOSO, 1995; FRIES, 1996). Além do conhecimento e da adequada utilização dos escores visuais, o sucesso de qualquer programa de melhoramento depende do conhecimento dos efeitos ambientais que afetam estas características.

O conhecimento dos parâmetros genéticos tem grande importância na determinação dos critérios de seleção que serão adotados no rebanho que se quer melhorar. Existe uma marcada diferença nestes parâmetros entre populações criadas em diferentes ambientes e também através do tempo. Por isto, é necessária a estimação adequada dos parâmetros genéticos para uma boa condução de programas de seleção de uma raça, além do que, poucos são os trabalhos que estimam herdabilidades para escores visuais para animais de raças taurinas no Brasil (MOREIRA \& CARDELLINO, 1994 e CARDOSO et al., 2001b).

O objetivo deste trabalho foi avaliar a influência de alguns fatores ambientais sobre os escores de avaliação visual à desmama e estimar parâmetros genéticos, para bezerros da raça Charolês, criados no Sul do Brasil.

\section{MATERIAL E MÉTODOS}

O presente estudo foi desenvolvido com dados coletados pelo Programa de Melhoramento de Bovinos de Carne - PROMEBO, executado pela Associação Nacional de Criadores "Herd Book Collares", em rebanhos da raça Charolês. Os dados foram coletados durante os anos de 1994 a 2002, em 17 fazendas nos estados do Rio Grande do Sul e Paraná.

Os escores visuais (EVs) conformação, precocidade, musculatura e tamanho, foram observados na ocasião da desmama dos animais. As notas não são atribuídas em uma escala absoluta como no sistema Ankony de LONG (1973). Desta forma, o julgador atribui ao grupo de contemporâneos (GC), nota três ao animal considerado detentor da média para a característica, nota um e cinco para os animais com menor e maior expressão da característica dentro daquele grupo, respectivamente. Portanto, as notas são atribuídas ao animal em relação ao seu GC.

Entende-se como precocidade $(\mathrm{P})$ a rapidez no acabamento da carcaça, quando o animal possuir as formas musculares desenvolvidas e reservas de gordura, porém sem um grande peso vivo; animais com maior profundidade de costelas, maior caixa torácica, de silhueta cheia, com virilhas pesadas e em início de 
deposição de gordura subcutânea, principalmente na base da cauda, indicam maior precocidade de terminação. Musculatura (M) é a presença de massas musculares principalmente no quarto traseiro, lombo, paleta e antebraço, sendo um escore que merece destaque pelos produtores que buscam selecionar animais adequados para os padrões requeridos pelos frigoríficos. Tamanho $(\mathrm{T})$ representa a interação entre altura e comprimento do animal. Já o escore de conformação (C) representa, de forma abrangente, a capacidade do animal em termos de produção de carne, se abatido naquele momento, considerando-se a combinação de comprimento e profundidade do corpo, desenvolvimento muscular e harmonia geral do indivíduo.

$\mathrm{O}$ arquivo original estava constituído de 12.868 animais, após a exclusão dos indivíduos que não possuíam escores à desmama registrados, restaram 2.372 observações para C, 2.041 para $\mathrm{P}, 2.251$ para $\mathrm{T}$ e 2.371 para M. Os grupos de contemporâneos foram formados pela concatenação de fazenda, sexo e ano de nascimento do animal. Foram eliminados os grupos de contemporâneos com menos de cinco observações e touros que possuíam menos de cinco filhos. A idade à desmama variou de 107 a 300 dias, a data juliana de nascimento teve como mínimo de 50 e o máximo de 361 dias e a idade ao parto mínima foi 1.095 (três anos) e a máxima foi 4.745 dias (treze anos). Após a editoração dos dados os mesmos foram submetidos a uma análise de variância, utilizando-se o programa SAS - Statistical Analysis System (SAS, 2001), para identificar os efeitos de ambiente que afetavam significativamente as características em estudo. Posteriormente, os efeitos significativos foram analisados de acordo com um modelo que considerou as características de conformação, precocidade, musculatura e tamanho em função do efeito fixo do grupo de contemporâneos $\left(\mathrm{GC}_{\mathrm{i}}\right)$ e das covariáveis idade do animal à desmama (ID $)_{\mathrm{j}}$, data juliana de nascimento $\left(\mathrm{DJ}_{\mathrm{k}}\right)$ e idade da vaca ao parto ( $\left(V_{1}\right)$, além do efeito residual, aleatório. Os escores visuais foram analisados separadamente, através do método dos Quadrados Mínimos pelo procedimento PROC GLM (SAS, 2001). Após, as médias dos quadrados mínimos obtidas para IV e DJ, foram utilizadas em análise de regressão quadrática em relação à característica estudada e a estes efeitos, explicando o efeito da idade da vaca ao parto e da data juliana de nascimento em relação aos escores visuais. As médias de quadrados mínimos da classe ID foram utilizadas em análise de regressão linear simples.

Para a estimação dos componentes de (co)variância, usados para estimar os parâmetros genéticos, os dados foram analisados pelo Método da Máxima Verossimilhança Restrita livre de derivada, através do algoritmo desenvolvido por BOLDMAN et al., (2001), disponível no aplicativo computacional MTDFREML.

Para essas análises o modelo geral utilizado pode ser representado na forma matricial como:

$$
\mathrm{Y}=\mathrm{X} \beta+\mathrm{Z}_{1 \mathrm{~d}}+\mathrm{Z}_{2} \mathrm{~m}+\varepsilon
$$

em que: $\mathrm{y}=$ vetor de variáveis dependentes (escores visuais); $\mathrm{X}=$ matriz de incidência que associa cada observação a cada efeito fixo; $\beta=$ vetor de efeitos fixos; $Z^{1}=$ matriz de incidência dos efeitos genéticos aditivos diretos; $\mathrm{d}=$ vetor dos efeitos genéticos aditivos diretos; $Z^{2}=$ matriz de incidência dos efeitos genéticos aditivos maternos; $\mathrm{m}=$ vetor dos efeitos genéticos aditivos maternos; $\varepsilon=$ vetor de efeitos residuais. Neste modelo, foi considerado como efeito fixo, o grupo de contemporâneos, além das covariáveis idade da vaca ao parto e data Juliana de nascimento (efeitos linear e quadrático) e idade a desmama (efeito linear).

\section{RESULTADOS E DISCUSSÃO}

As médias e os desvios padrões observados para C, P, M e T foram, respectivamente, 3,65 $(0,99)$; $3,42(1,10) ; 3,48(1,10)$ e 3,64 (1,06) unidades de escores visuais, que estão adequadas, uma vez que as notas são conferidas em relação à média do grupo contemporâneo em que o animal foi avaliado.

A idade média das vacas ao parto, neste trabalho, foi 6,52 anos, maior do que aquela relatada por JORGE JÚNIOR et al. (2001), para matrizes da raça Nelore, que foi de 5,5 anos.

Todos os efeitos fixos considerados no modelo foram significativos para os escores avaliados, exceto a data Juliana, efeitos linear e quadrático, para $\mathrm{M}, \mathrm{P}$ e C e idade à desmama, efeito quadrático para as quatro características estudadas (Tabela 1). Estes resultados concordam com PONS et al. (1989), que encontraram efeito significativo da idade da mãe ao parto e da estação de nascimento, para o escore de conformação, em bovinos da raça Hereford criados no Rio Grande do Sul e também com JORGE JÚNIOR et al., (2001) para a raça Nelore.

Na tabela 2, estão apresentadas as equações de predição de $\mathrm{C}, \mathrm{M}, \mathrm{P}$ e T em relação à data juliana de nascimento (DJ), idade da vaca (IV) e idade do bezerro à desmama (ID) e, para uma melhor compreensão, foram estimadas curvas que representam o efeito ambiental em questão sobre o escore visual analisado (Figura 1).

Com relação à DJ, verifica-se que os escores visuais (EVs) cresceram até o mês de junho, decrescendo em seguida, atingindo os menores valores em dezembro, com maior redução para $\mathrm{T}$, seguido por $\mathrm{C}$ 
Tabela 1 - Fontes de variação, graus de liberdade (GL) e quadrados médios para os escores de musculatura (M), precocidade (P), conformação (C) e tamanho(T).

\begin{tabular}{|c|c|c|c|c|c|c|c|c|}
\hline \multirow[t]{2}{*}{ Fontes de variação } & \multicolumn{4}{|c|}{ GL } & \multicolumn{4}{|c|}{ Quadrados médios } \\
\hline & M & $\mathrm{P}$ & $\mathrm{C}$ & $\mathrm{T}$ & M & $\mathrm{P}$ & $\mathrm{C}$ & $\mathrm{T}$ \\
\hline Grupo contemporâneo & 66 & 65 & 66 & 63 & $11.1078^{* *}$ & $13.5518^{* *}$ & $8.7631^{* *}$ & $9,8970^{* *}$ \\
\hline \multicolumn{9}{|l|}{ Data Juliana } \\
\hline Linear & 1 & 1 & 1 & 1 & $1.2258^{\mathrm{NS}}$ & $2.3093^{\mathrm{NS}}$ & $2.2964^{\mathrm{NS}}$ & $15,6089^{* *}$ \\
\hline Quadrático & 1 & 1 & 1 & 1 & $1.2258^{\mathrm{NS}}$ & $2.2603^{\mathrm{NS}}$ & $3.4709^{\mathrm{NS}}$ & $20,8862^{* *}$ \\
\hline Idade desmama & 1 & 1 & 1 & 1 & $3.8127^{*}$ & $13.4165^{* *}$ & $3.0691^{* *}$ & $10,1124^{* *}$ \\
\hline \multicolumn{9}{|l|}{ Idade da vaca } \\
\hline Linear & 1 & 1 & 1 & 1 & $89.5788^{* *}$ & $64.7208^{* *}$ & $74.7631^{* *}$ & $86,0219^{* *}$ \\
\hline Quadrático & 1 & 1 & 1 & 1 & $71.4097^{* *}$ & $53.1167^{* *}$ & $61.8001^{* *}$ & $72,9176^{* *}$ \\
\hline Erro & 2.300 & 1.975 & 2300 & 2.182 & 0.8095 & 0.6938 & 0.6715 & 0.6921 \\
\hline $\mathrm{R}^{2}$ & & & & & 0.3520 & 0.4478 & 0.3438 & 0.3994 \\
\hline
\end{tabular}

$* *(\mathrm{P}<0,001) ; *(\mathrm{P}<0,05) ;{ }^{\text {NS }}$ (Não significativo)

e M. Para P, o comportamento foi semelhante, mas os menores valores (Figura 1), observados em dezembro, ainda foram superiores aos verificados em janeiro, mantendo, portanto, tendência de crescimento com a data juliana. Este comportamento pode ser explicado, em parte, pelas condições climáticas encontradas neste período. Os animais nascidos no mês de junho tiveram a maior parte do seu desenvolvimento durante a primavera e o verão, estações estas, propícias para o crescimento das pastagens, favorecendo a produção de leite das mães e refletindo no desenvolvimento do bezerro, pois nesta fase da vida, o mesmo depende da mãe para suprir suas exigências nutricionais. Da mesma forma, os animais nascidos em dezembro e janeiro, receberam menores escores visuais, em conseqüência de terem sido desmamados em junho e julho e de terem passado boa parte do seu desenvolvimento nas estações de outono e inverno. Tendência semelhante foi relatada por CARDOSO et al. (2001a), os quais verificaram, para a raça Angus, maiores EVs para os bezerros nascidos nos meses de julho e agosto e desmamados nos meses de primavera-verão, época em que as pastagens apresentam melhor qualidade.

Com relação à IV, verifica-se que os escore visuais foram maiores para os filhos de vacas com idade entre sete e oito anos, para as quatro características

Tabela 2 - Coeficientes das equações de regressão para conformação, precocidade, musculatura e tamanho a desmama, em função da data juliana de nascimento, idade da vaca ao parto e idade a desmama.

\begin{tabular}{|c|c|c|c|}
\hline \multirow[b]{2}{*}{ Efeito/Escore visual } & \multicolumn{3}{|c|}{ Parâmetro } \\
\hline & Intercepto & Linear & Quadrático \\
\hline \multicolumn{4}{|c|}{ Data juliana de nascimento (dias) } \\
\hline Conformação & 2,2996 & 0,0181 & $-0,00005$ \\
\hline Tamanho & 1,9844 & 0,0234 & $-0,00006$ \\
\hline Precocidade & 1,4003 & 0,0250 & $-0,00006$ \\
\hline Musculatura & 1,9106 & 0,0202 & $-0,00005$ \\
\hline \multicolumn{4}{|c|}{ Idade da vaca ao parto (anos) } \\
\hline Conformação & 2,7989 & 0,2609 & $-0,0176$ \\
\hline Tamanho & 2,8116 & 0,2688 & $-0,0192$ \\
\hline Precocidade & 2,7497 & 0,2078 & $-0,0142$ \\
\hline Musculatura & 2,4815 & 0,2973 & $-0,0195$ \\
\hline \multicolumn{4}{|c|}{ Idade à desmama (dias) } \\
\hline Conformação & 2,8528 & 0,0036 & \\
\hline Tamanho & 2,5102 & 0,0051 & \\
\hline Precocidade & 2,6956 & 0,0032 & \\
\hline Musculatura & 2,7844 & 0,0032 & \\
\hline
\end{tabular}



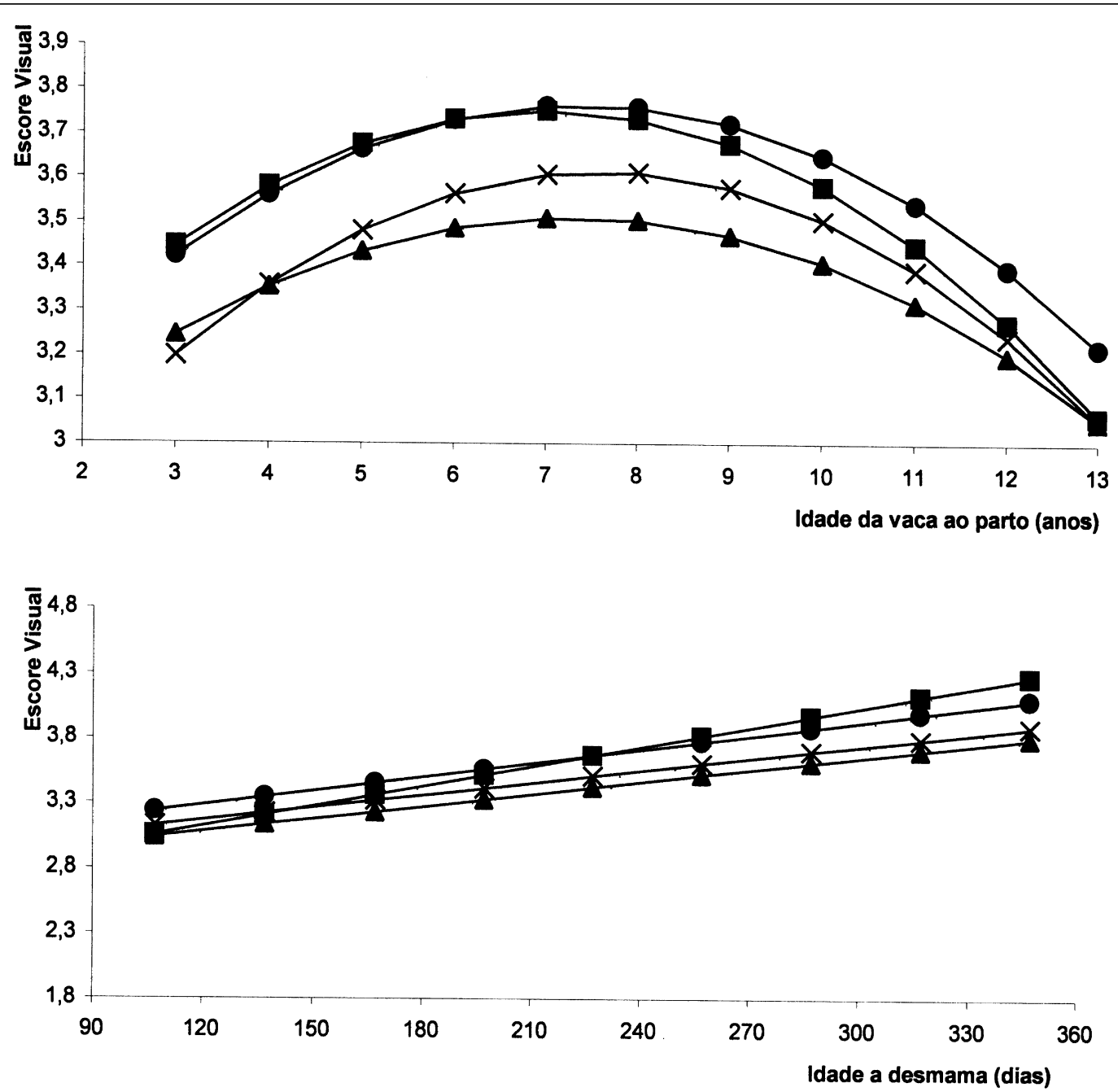

2

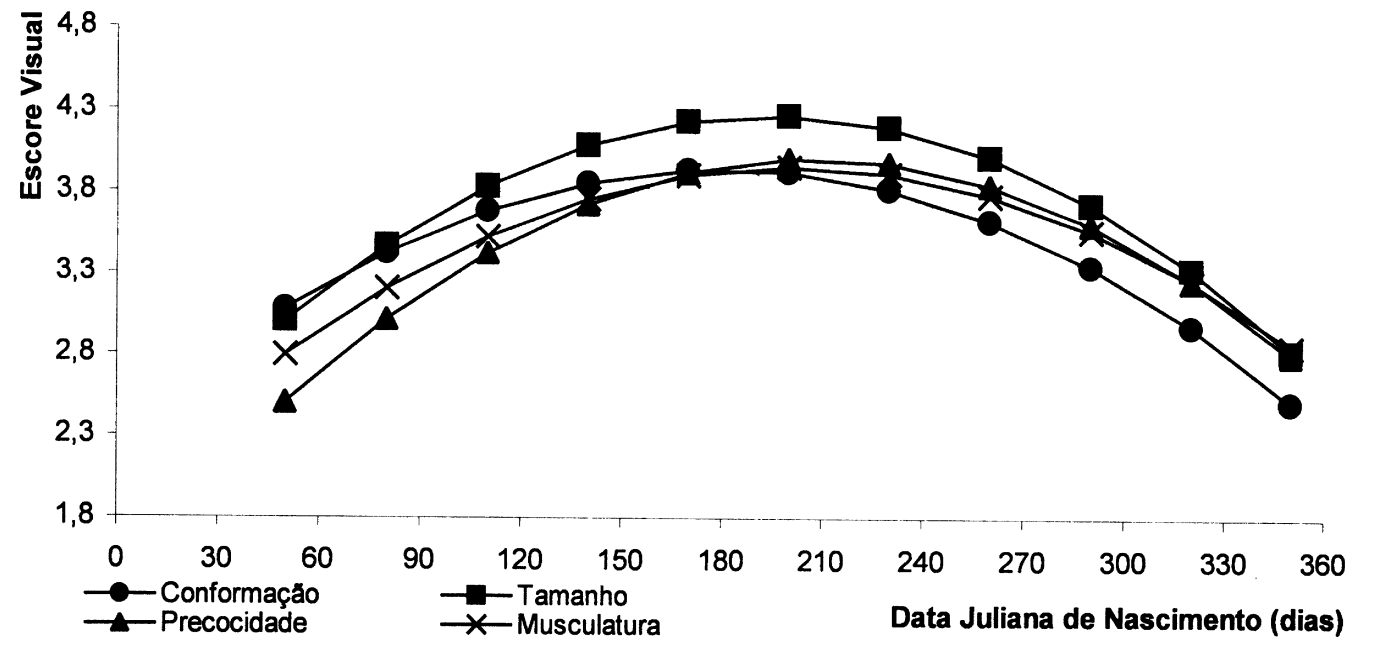

Figura 1 - Curvas estimadas para conformação, precocidade, musculatura e tamanho à desmama, de acordo com os fatores ambientais estudados.

Ciência Rural, v.36, n.2, mar-abr, 2006. 
estudadas, decrescendo à medida que as mães foram se tornando mais velhas. Para PONS et al. (1989), para a raça Hereford, o efeito da idade da vaca sobre o escore de conformação do filho à desmama, teve efeito quadrático. Terneiros filhos de vacas jovens, até três anos e com idades superiores a treze anos tiveram influência acentuada no escore de conformação. Relação semelhante a esta também foi relatada por DAL-FARRA et al. (2002) que, para animais Nelore $x$ Hereford, observaram uma melhora no desempenho dos bezerros com o aumento da idade da vaca, atingindo o pico por volta dos sete anos de idade, decrescendo gradativamente a partir dessa idade. JORGE JÚNIOR et al. (2001) e CARDOSO et al. (2001a) relataram aumento nos escores visuais com a idade da vaca até a mesma atingir sete e oito anos de idade, decrescendo a seguir, para rebanhos da raça Nelore e Angus, respectivamente.

Com relação ao efeito da idade à desmama, verifica-se que os EVs cresceram em função desta para as quatro características estudadas. Esta tendência pode ser um reflexo da idade do animal, ou seja, animais mais velhos tendem a serem maiores e, também, mais pesados, isto talvez confunda o avaliador, que emite uma melhor pontuação para estes animais, tal como proposto por JORGE JÚNIOR et al. (2001).

De um modo geral, observa-se que os mesmos fatores que afetam o peso à desmama, nfluenciam também os escores visuais. Esta constatação foi obtida por MOREIRA \& CARDELLINO (1994), que, trabalhando com bezerros Hereford, observaram que as médias dos escores de conformação, praticamente acompanharam o comportamento das médias dos pesos à desmama. Tal comprovação também foi relatada por DAL-FARRA et al. (2002) que, em estudo com bezerros das raças Hereford, Nelore e do cruzamento entre elas, concluíram que a idade do bezerro, a idade da vaca e a data juliana do nascimento do bezerro, são importantes fontes de variação nos escores de conformação, precocidade e musculatura a desmama, similarmente ao verificado para ganho de peso do nascimento a desmama.

As estimativas dos componentes de variância e parâmetros genéticos para C, P, M e T, obtidas neste trabalho, estão apresentadas na tabela 3 . Nela, pode-se verificar que os coeficientes de herdabilidade variaram de 0,27 a 0,37 (direta) e de 0,19 a 0,29 (materna). Os valores das estimativas de herdabilidade direta, neste trabalho, foram maiores do que aqueles estimados por dois métodos (REML e método R) por VAN MELLIS et al. (2003), para raça Nelore $(0,22$ e 0,21 para $C ; 0,21$ e 0,21 para $\mathrm{Pe} 0,22$ e 0,22 para M); por BALBÉ et al. (2003), para a raça Brangus, para C, P e M, respectivamente, 0,$23 ; 0,34$ e 0,23 e por CARDOSO et al. (2001b), para a raça Angus, 0,15; 0,18; 0,17 e 0,18 respectivamente para C, P, M e T. Ressaltase que as estimativas de herdabilidade obtidas neste trabalho, podem estar sendo afetadas pela inconsistência no sistema de avaliação, variação entre avaliadores, ou mesmo, devido a diferenças genéticas

Tabela 3 - Estimativas dos componentes de (co)variância e parâmetros genéticos para conformação (C), musculatura (M), precocidade (P) e tamanho $(\mathrm{T})$ à desmama, a partir de análises univariadas.

\begin{tabular}{lllll}
\hline Características & $\mathrm{C}$ & $\mathrm{M}$ & $\mathrm{P}$ & $\mathrm{T}$ \\
\hline Componentes de variância & & & & \\
$\delta^{2}$ & & & & 0,20 \\
$\delta_{\mathrm{a}}^{2}$ & 0,25 & 0,29 & 0,17 & 0,14 \\
$\delta_{\mathrm{am}}$ & 0,20 & 0,24 & $-0,15$ & $-0,11$ \\
$\delta_{\mathrm{e}}^{2}$ & $-0,19$ & $-0,22$ & 0,46 & 0,49 \\
$\delta_{\mathrm{p}}^{2}$ & 0,43 & 0,53 & 0,72 & 0,72
\end{tabular}

Parâmetros genéticos

$\begin{array}{lcccc}\mathrm{h}^{2}{ }_{\mathrm{a}} & 0,37 \pm 0,09 & 0,35 \pm 0,09 & 0,33 \pm 0,09 & 0,27 \pm 0,09 \\ \mathrm{~h}^{2}{ }_{\mathrm{m}} & 0,29 \pm 0,08 & 0,28 \pm 0,08 & 0,24 \pm 0,09 & 0,19 \pm 0,08 \\ \mathrm{r}_{\mathrm{am}} & -0,86 \pm 0,12 & -0,85 \pm 0,11 & -0,73 \pm 0,18 & -0,66 \pm 0,20 \\ \mathrm{e}^{2} & 0,63 \pm 0,07 & 0,63 \pm 0,07 & 0,64 \pm 0,07 & 0,68 \pm 0,07\end{array}$

$\delta_{\mathrm{a}}^{2}=$ variância genética aditiva direta, $\delta_{\mathrm{m}}^{2}=$ variância genética aditiva materna, $\delta_{\mathrm{am}}=$ covariância genética entre os efeitos aditivos direto e materno, $\delta_{\mathrm{e}}^{2}=$ variância ambiental, $\delta_{\mathrm{p}}^{2}=$ variância fenotípica. $\mathrm{h}_{\mathrm{a}}^{2}=$ herdabilidade aditiva direta, $\mathrm{h}_{\mathrm{m}}^{2}=$ herdabilidade aditiva materna, $\mathrm{r}_{\mathrm{gm}}=$ correlação genética entre os efeitos aditivos direto e materno e $\mathrm{e}^{2}=$ porção da variância devido ao ambiente temporário 
naturais entre as populações, tal como citado por CARDOSO et al. (2004). Mesmo assim, é necessário que avaliações com conjuntos de dados maiores sejam realizadas para esta raça, e que a variância ambiental seja reduzida, através de melhor controle do ambiente (alimentação, grupo de contemporâneos, condições sanitárias, treinamento dos avaliadores, etc.). Em assim procedendo, estariam sendo criadas condições que permitirão obter-se estimações mais acuradas para os parâmetros genéticos para os escores visuais.

\section{CONCLUSÕES}

Os dados analisados permitem concluir que os escores visuais estudados são afetados pelos efeitos de meio e, estes, devem ser considerados nos modelos de análise, nos programas de seleção para a raça Charolês.

As estimativas de herdabilidade direta para os escores visuais de conformação, precocidade, musculatura e tamanho, obtidas, neste estudo permitem concluir que a seleção para estas características poderá promover algum ganho genético.

Com a possibilidade da inserção destas características nos critérios de seleção, em bovinos de corte e considerando a relevância do tema, sugere-se que mais estudos, com maior volume de dados, sejam realizados, para esta raça.

\section{AGRADECIMENTOS}

À Coordenação de Aperfeiçoamento de Pessoal de Nível Superior (CAPES), pela concessão da bolsa ao pesquisador Kippert.

Ao Programa Institucional de Bolsas de Iniciação Científica, Conselho Nacional de Desenvolvimento Científico e Tecnológico (PIBIC, CNPq) pela concessão da bolsa ao pesquisador Boligon

\section{REFERÊNCIAS}

BALBÉ, D.D. et al. Estimativas de herdabilidade para os escores visuais de conformação, precocidade e musculatura, em rebanhos da raça Brangus. In: REUNIÃOANUALDASOCIEDADE BRASILEIRA DE ZOOTECNIA, 40., 2003, Santa Maria - RS. Anais... Santa Maria: Sociedade Brasileira de Zootecnia, 2003. 1 CD.

BOLDMAN, K.G. et al. A manual for use of MTDFREML. A set of programs to obtain estimates of variances and covariances. (Draft). Lincoln: Department of Agriculture, Agricultural Research Service, 2001, 117p.

CAMPOS, L.T.; CARDOSO, F.F. Programa de melhoramento de bovinos de carne. Manual do usuário. Pelotas-RS: Associação Nacional de Criadores "Herd Book Collares", 1995, $79 \mathrm{p}$

CARDOSO, F.F. et al. Fatores ambientais sobre escores de avaliação visual à desmama em bezerros Angus criados no Rio
Grande do Sul. Revista Brasileira de Zootecnia, v.30, n.2, p.318-325, 2001a.

CARDOSO, F.F. et al. Componentes de (Co)variância e parâmetros genéticos para caracteres produtivos à desmama de bezerros Angus Criados no Estado do Rio Grande do Sul. Revista Brasileira de Zootecnia, v.30, n.1, p.41-48, 2001 b.

CARDOSO, F.F. et al. Componentes de (Co)variância e parâmetros genéticos para caracteres produtivos pós-desmama em bovinos da raça Angus. Revista Brasileira de Zootecnia, v.33, n.2, p.313-319, 2004

DAL-FARRA, R. et al. Efeitos de ambiente e de heterose sobre o ganho de peso do nascimento ao desmame e sobre os escores visuais ao desmame de bovinos de corte. Revista Brasileira de Zootecnia, v.31, n.3, p.1350-1361, 2002 (suplemento).

FERREIRA, G.B. et al. Variance components and breeding values for growth traits from different statistical models. Journal of Animal Science, v.77, p.2641-2650, 1999.

FRIES L.A. Uso de escores visuais em programas de seleção para a produtividade em gado de corte. In: SEMINÁRIO NACIONAL - REVISÃO DE CRITÉRIOS DE JULGAMENTO E SELEÇÃO EM GADO DE CORTE, 1996, Uberaba-MG, Anais... Uberaba: ABCZ, 1996. p.1-6.

JORGE JÚNIOR, J. et al. Influência de alguns fatores de ambiente sobre os escores de conformação, precocidade e musculatura a desmama em um rebanho da raça Nelore. Revista Brasileira de Zootecnia, v.30, n.6, p.1697-1703, 2001

KIPPERT, C.J. et al. Tendência genética para características de peso em bovinos da raça Charolês no Estado do Rio Grande do Sul. In: REUNIÃO ANUAL DA SOCIEDADE BRASILEIRA DE ZOOTECNIA, 41., 2004, Campo Grande-MS. Anais... Campo Grande, MS: Sociedade Brasileira de Zootecnia, 2004. 1 CD.

LONG, R.A. El sistema de evaluation de Ankony y su aplicación en la mejora del ganado. Colorado: Ankony Corporation, Grand Juntion, 1973. 21p.

MOREIRA, H.L.M.; CARDELLINO R.A. Herdabilidade, repetibilidade e efeitos ambientais em bovinos Hereford. Pesquisa Agropecuária Brasileira, v.29, n.11, p.1795-1800, 1994.

NRC - NATINAL RESEARCH COUNCIL. Nutrient requeriments of beef cattle. Washington, DC: National Academy of Sciences, 1996. 242p.

PONS, S.B. et al. Efeitos de fatores genéticos e de ambiente sobre o crescimento e o escore de conformação em bovinos Hereford no Rio Grande do Sul. I - Peso e escore de conformação à desmama. Revista Brasileira de Zootecnia, v.18, n.5, p.391-401, 1989

SAS INSTITUTE INC. SAS/STAT. User's Guide: stat. Release 8.1 Edition. Cary, 2001, 1292p.

VAN MELIS, M.H. et al. estimação de parâmetros genéticos em bovinos de corte utilizando os métodos de máxima verossimilhança restrita e R. Revista Brasileira de Zootecnia, v.32, n.6, p.1624-1632, 2003 